\title{
超声场中高分子乳液稳定性与多孔材料结构的调控机理研究
}

\author{
朱先念陈芳翟薇* 魏炳波 \\ (西北工业大学理学院 西安 710129)
}

\begin{abstract}
摘要 采用星型聚合物 POSS-(PMMA) 8 作为乳化剂兼成膜基质, 使用超声乳化法制备 w/o 型聚合物乳液, 制备的乳液 液滴初始直径受超声功率调控, 阐明了超声功率对液滴直径大小的作用规律. 结果表明乳液液滴直径呈双峰分布, 且 随超声功率的增大液滴直径增大. 通过改变水相离子浓度制备聚合物乳液, 得到的乳液液滴直径 $10 \mu \mathrm{m}$ 左右且单分散 性好, 并发现随超声功率增大乳液的絮凝程度增大. 探索了超声功率对乳液失稳机制的影响, 当超声功率低于 $750 \mathrm{~W}$ 时，奥氏熟化是乳液失稳的主要机制. 通过浸渍法制备以玻璃纤维为基底的多孔膜材料，发现多孔材料的孔径变化与 对应乳液的液滴直径变化基本一致. 本研究证实了通过改变超声功率可以调控乳液的液滴直径, 然后利用乳液模板法 能获得结构和形貌可控的多孔材料.
\end{abstract}

关键词 超声; 乳化; 高分子乳液; 乳液模板法; 多孔材料

\section{Modulation Mechanisms of Polymeric Emulsion Stability and Porous Material Architecture within Ultrasonic Fields}

\author{
Zhu, Xiannian Chen, Fang Zhai, Wei* Wei, Bingbo \\ (School of Natural and Applied Sciences, Northwestern Polytechnical University, Xi'an 710129)
}

\begin{abstract}
Ultrasonic emulsification has received great attention recently due to the great potential of tuning the emulsion droplets size compared to the traditional homogeneous mixing method. In this study, a series of polymeric emulsions were prepared by ultrasonic emulsification approaches. The relationships between ultrasonic power, emulsion destabilization mechanism and structure morphology of macroporous polymer material were studied. The water in toluene emulsion was prepared by mixing the polymer POSS-(PMMA) $)_{8}$ toluene solution $\left(10 \mathrm{mg} \bullet \mathrm{mL}^{-1}\right)$ and water or $\mathrm{Na}_{2} \mathrm{CO}_{3}$ aqueous solution $(0.1$ $\left.\mathrm{mol} \cdot \mathrm{L}^{-1}\right)$ through different ultrasonic powers. The volume ratio of water/toluene was remained as $50: 50$. The evolution of emulsion droplets diameter distribution over time was analyzed based on optical microscopy data. The initial droplets diameter increased with the increase of ultrasonic power, and all emulsion droplets diameter distributions followed the diauxie curve. By changing the ion concentration in aqueous solution, excellent monodisperse emulsions with droplets diameter around 10 microns were acquired. It was found out that flocculation degree of emulsions prepared with $\mathrm{Na}_{2} \mathrm{CO}_{3}$ aqueous solution was increased with increasing of ultrasonic power. The relationship between ultrasonic power and emulsion stability was clarified by fitting the emulsion droplets diameter variation as the function of time. When ultrasonic power was below $750 \mathrm{~W}$, Ostwald ripening dominated the instability mechanism of emulsion. Once the ultrasonic power achieved to $1000 \mathrm{~W}$, the instability mechanism of emulsion was attributed to Ostwald ripening and coalescence at the same time. The macroporous polymer materials with adjustable pore diameter were prepared by dipping the glass fiber into salt containing emulsions. In this approach, POSS-(PMMA) 8 not only acted as emulsifier during emulsion preparation, but also treated as the skeleton of porous materials. The droplet-size of emulsions was monitored by changing ultrasonic power, meanwhile the structure and morphology of porous materials could be controlled on the basis of emulsion template.

Keywords ultrasonic field; emulsification; polymeric emulsion; emulsion template; porous material
\end{abstract}

\section{1 引言}

乳液是由两个互不相溶的液相组成, 采用乳化剂维 持稳定的一相以液滴的形式分散在另一相中的混合体 系，被广泛地应用于众多领域 ${ }^{[1]}$. 此外，近年来利用乳
液作为模板制备微纳米材料 ${ }^{[2]}$, 由于方法简单易行、可 规模制备等特点，在储氢、吸附 ${ }^{[3]}$ 、催化等方面有诸多 应用. 如可通过制备的 w/o 型 ${ }^{[4]}$ 和 $\mathrm{o} / \mathrm{w}$ 型乳液 ${ }^{[5]}$ 作为模板 制备不同孔结构的多孔结构材料和聚合物微球. 其中, 乳液液滴直径大小直接影响到多孔材料的孔径, 因此在

\footnotetext{
*E-mail: zhaiwei322@nwpu.edu.cn; Tel.: 15114816416

Received March 13, 2017; published June 26, 2017.
}

Supporting information for this article is available free of charge via the Internet at http://sioc-journal.cn.

Project supported by the National Natural Science Foundation of China (Nos. 51327901 and 51571164), Science and Technology New Star Project of Shaanxi Province (No. 2016KJXX-85), Surface of the Shaanxi Province Natural Science Fund Project (No. 2016JM2023), Shaanxi Provincial Key Laboratory of Science and Technology Innovation Project.

国家自然科学基金(Nos. 51327901, 51571164)、陕西省科技新星项目(No. 2016KJXX-85)、陕西省自然科学基金面上项目(No. 2016JM2023)以及陕西省 科技统筹创新工程重点实验室资助项目. 
乳液模板法制备多孔材料的过程中关键问题是如何调 控乳滴直径、探索乳液的失稳机制.

一般来说, 乳液液滴直径越小, 其稳定存在的时间 越长, 从而具有较大的应用价值. 目前普遍采用添加传 统表面活性剂在剪切场下制备乳液的方法, 该方法存在 液滴直径大、单分散性差的缺点. 超声场作为一种有效 的调控手段, 得到了许多研究者的关注 ${ }^{[6]}$. 超声乳化方 法主要是利用超声空化效应在液体内部产生局部的高 温高压和微射流, 使两相混合实现乳化. 较之于一般的 手摇法、均质摚拌法等方法有着乳化剂用量少、液滴直 径小、稳定性好的优点 ${ }^{[7]}$. 魏晓莉等 ${ }^{[8]}$ 通过超声乳化法制 备以 Tween 80 等离子型表面活性剂为乳化剂的乳化柴 油, 发现随超声功率的增大, 乳化柴油的稳定性逐渐增 加; 而超声作用时间为 $15 \mathrm{~min}$ 最佳, 时间过短乳化不完 全, 时间过长则分散相会重新凝聚. Sui 等 ${ }^{[9]}$ 通过超声处 理卵磷脂稳定的乳化体系, 发现随超声功率的增加, 乳 液的稳定性减弱, 超声功率为 $150 \mathrm{~W}$ 时乳液显现最佳稳 定性. Leong 等 ${ }^{[10]}$ 通过超声乳化制备 Tween80 和 Span80 乳化剂稳定的葵花油/水微乳液体系, 并分析超声辐射 能量、声压细化微乳液液滴直径的作用规律, 实验制备 的微乳液液滴直径小于 $40 \mathrm{~nm}$.

但是, 超声乳化法的研究大多集中于超声在传统表 面活性剂 ${ }^{[11]}$ 乳液体系中的影响机制, 而对于功率超声在 制备大分子乳液过程中的作用机制还鲜有报道. 本文在 频率 $f=20 \mathrm{kHz}$ 的功率超声下使用实验室自制的聚合物 POSS-(PMMA) ${ }_{8}{ }^{[12]}$ 作为乳化剂兼成膜物质制备高分子 乳液, 研究了超声功率对乳液液滴直径、乳液失稳机制 的作用规律. POSS-(PMMA) 8 具有星型拓扑结构, 以其 作为乳化剂制备油包水的乳液, 不仅可以避免表面活性 剂的应用, 且利用其良好的成膜性, 可以通过超声功率 调控乳液液滴粒径及分布的手段, 以此调控 POSS-(PMMA) 8 为骨架的多孔材料的孔径及分布, 以期
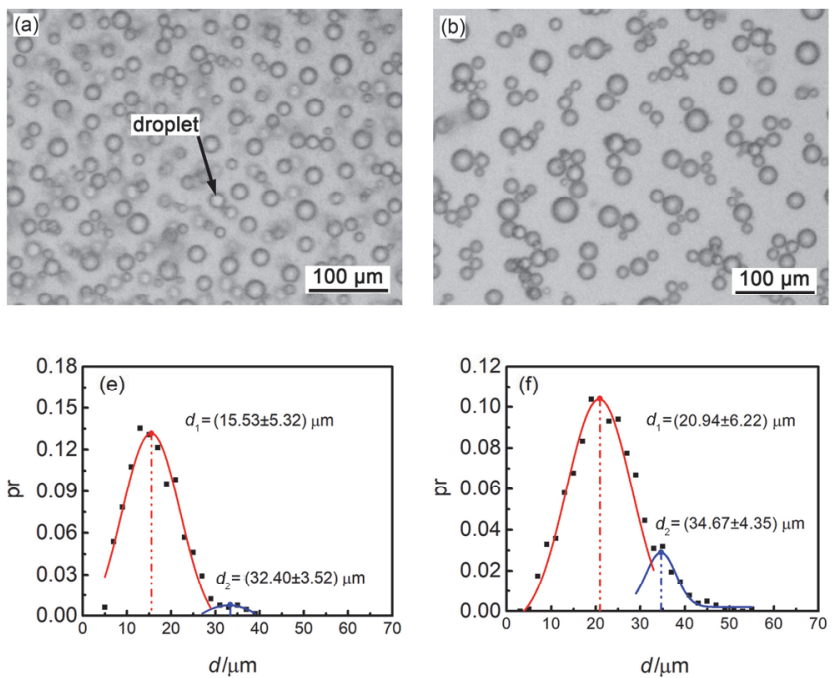

获得性质稳定、孔径可控的聚合物多孔材料.

\section{2 结果与讨论}

\section{1 高分子乳液分散相直径随超声功率的变化特征}

图 1 给出了不同超声功率作用下 $w / 0$ 型乳液的微观 形貌和对应的液滴直径分布图. 实验选取四个不同超声 功率 $(250 、 500 、 750$ 和 $1000 \mathrm{~W})$, 作用于水与甲苯体积 比为 $1: 1$ 的样品中 $5 \mathrm{~min}$, 在乳液制备完成 $1 \mathrm{~h}$ 内通过 光学显微镜观察其微观形貌(图 $1 \mathrm{a} \sim 1 \mathrm{~d}$ ), 此时观察到的 液滴直径可视为液滴初始直径. 随超声功率的增加, 液 滴直径逐渐增大，且不同超声功率下各个样品的液滴直 径大小均分布在两个不同的直径区间.

进一步对每个样品的液滴直径分布进行统计, 得到 直径分布图(图 1e 1h). 从液滴直径分布图更明显看出 样品液滴直径在两个不同的区间分布. 对液滴直径统计 数据做高斯分布拟合, 得到每组样品平均直径. 由统计 数据得出, 随超声功率增加样品的液滴直径随之增大. 乳液平均直径由 $P=250 \mathrm{~W}$ 时的最小值 $d_{\min }=16.53 \mu \mathrm{m}$, 增加到 $P=1000 \mathrm{~W}$ 时的最大值 $d_{\text {max }}=30.08 \mu \mathrm{m}$. 由图 $2 \mathrm{a}$ 乳液平均直径随超声功率变化曲线可以看出, 高斯分布 拟合的峰值代表每个直径分布区间的平均值, 随超声功 率的增加, 第一和第二个直径分布的平均值均随之增 大.

液滴直径随超声功率增加而增大主要是由于超声 作用影响了体系的粘度、水/甲苯界面张力和剪切速率. 乳液制备 $1 \mathrm{~h}$ 内的直径分布可视为初始直径, 符合 Taylor 提出的理论计算公式 ${ }^{[13]}$.

$$
d \propto \gamma / \eta \mu
$$

上式中 $\gamma$ 为乳液两相界面张力, $\eta$ 为连续相的粘度, $\mu$ 为 乳液制备时的剪切速率. 随超声功率的增加, 体系温度 明显上升。通过对实验中样品体系温度的测定, 发现
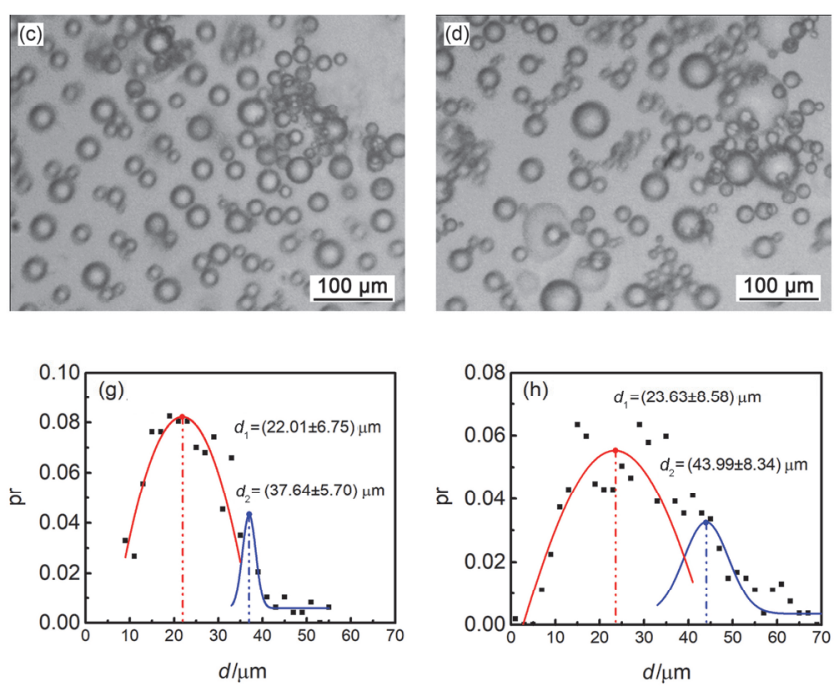

图 1 不同超声功率下制备的乳液初始状态下光学显微图和粒径分布图

Figure1 Microscope images and droplets size distribution of initial status emulsions prepared by different ultrasonic powers (a, e) $250 \mathrm{~W}$; (b, f) $500 \mathrm{~W}$; (c, g) $750 \mathrm{~W}$; (d, h) $1000 \mathrm{~W}$ 

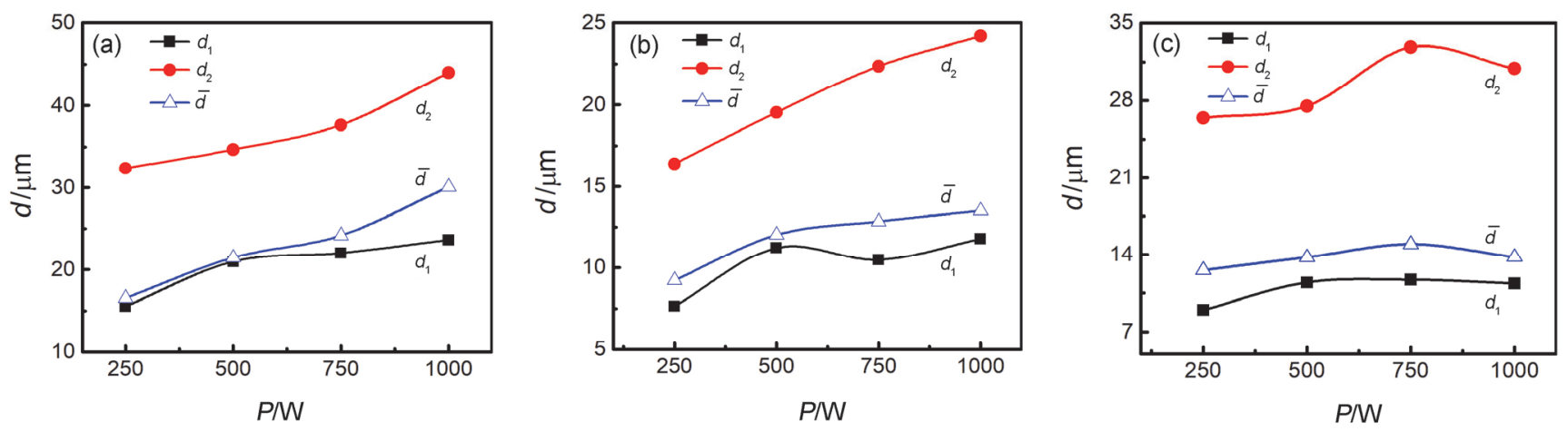

图 2 乳液平均粒径随超声功率变化曲线图

Figure 2 The average particle size of emulsion versus ultrasonic power

(a) initial status of salt free emulsions; (b) initial status of salt containing emulsions; (c) salt containing emulsions after $168 \mathrm{~h}$

$P=250 \mathrm{~W}$ 和 $P=1000 \mathrm{~W}$ 超声功率作用下样品温度相差 约 $30^{\circ} \mathrm{C}$. 体系温度上升使连续相粘度明显下降, 且温度 的上升也促进了聚合物分子的热运动, 使其在水/甲苯 界面吸附量减少, 增大了界面张力. 较大的超声功率下 空化效应产生的微射流流速更快, 超声作用下样品整体 的流场也有较快的流速, 这些因素为聚合物分子提供更 大动量, 促进聚合物分子从两相界面脱附. 由式(1)可知 连续相粘度越小和界面张力越大, 制备乳液的液滴直径 越大. 而超声功率增加导致剪切速率的改变对直径大小 的作用效果没有其它两相明显, 最终表现为随超声功率 的增加, 液滴直径随之增大.

而液滴直径呈双峰分布的原因是由于超声空化在 乳液中并不是均匀的作用于每个区域. 在空化效应没有 作用到的区域, 主要依靠超声作用下乳液整体流动提供 剪切力, 形成的液滴直径较大; 在空化效应作用区域内, 空化效应产生的高温高压及微射流将液滴进一步打碎, 产生更小的乳液液滴, 最终表现为液滴直径存在两个大 小不同的直径分布.

\section{2 超声乳化的含盐高分子乳液失稳机制}

在水相中加入电解质制备乳液, 能够提高乳液的稳 定性, 延长存放时间, 有助于进一步研究超声乳化和乳 液在其它方面的应用. 实验在不同超声功率下制备了以 $0.1 \mathrm{~mol} \cdot \mathrm{L}^{-1}$ 的 $\mathrm{Na}_{2} \mathrm{CO}_{3}$ 溶液为水相的乳液, 图 $3 \mathrm{a} \sim 3 \mathrm{~h}$ 为 乳液静置 $1 \mathrm{~h}$ 内在光学显微镜图和对应的直径分布图. 对比图 1 与图 3a～3h, 含盐乳液与不含盐乳液液滴直径 分布相似, 其直径分布有两个峰值且随超声功率增加液 滴直径随之增大.

从图 $2 a 、 2 b$ 含盐乳液与不含盐乳液平均直径之间 对比可知, 同一超声功率下含盐乳液的液滴直径与不含 盐乳液的液滴直径相比明显减小. 这是由于带电盐离子 的静电屏蔽作用降低了聚合物分子之间的静电斥力, 使 聚合物分子在界面吸附量增加, 降低了界面张力从而使 液滴直径减小. 此外, 含盐乳液中出现了不同程度的絮 凝, 且絮凝程度随超声功率的增加而增加. 据推测引起
这种变化的主要原因是因为不同超声功率下体系内电 荷分布情况存在差异, 引起了聚合物分子絮凝程度的不 同.

对比图 2b、2c 含盐乳液静置不同时间的平均液滴 直径变化, 随静置时间的增长液滴直径增大. 但不同超 声功率下制备的液滴直径增大程度不同. 乳液液滴长大 的过程主要有熟化和聚并 ${ }^{[14]}$ 两种形式. 根据 LSW (The Lifshitz-Slezov and Wagner)理论 ${ }^{[15]}$, 奥氏熟化的速率 $\omega$ 可以由下式表示:

$$
\omega=\partial \overline{d^{3}} / \partial t=C_{\infty} \gamma V_{m} D_{L} / 9 \rho R T
$$

其中 $\bar{d}$ 为液滴平均直径, $C_{\infty}$ 为无限大液滴在体相中的溶 解度, $\gamma$ 为界面张力, $V_{m}$ 为分散相的摩尔体积, $D_{L}$ 为分散 相在连续相中的扩散系数, $\rho$ 为分散相密度, $R$ 为气体常 数, $T$ 为绝对温度. 可见对于一个固定的体系, 仅考虑熟 化对液滴直径的作用, $\overline{d^{3}} \sim t$ 关系图应为一条直线, 且 斜率为熟化速率.

含盐乳液的液滴直径按上述方式拟合, 得到如图 4a 所示的直径 3 次方与时间的关系图. 可见当超声功率 低于 $750 \mathrm{~W}$ 时, 液滴直径变化趋势符合奥氏熟化的拟 合，说明奥氏熟化在液滴直径增大过程中起主导作用， 且随超声功率的增加奥氏熟化的速率增大. 这是由于在 固定成分的体系中, 仅改变超声功率, 对除界面张力 $\gamma$ 外其它物理量没有影响. 前文分析认为随超声功率的增 加, 乳液的界面张力随之增大. 式(2)中可以看出, 其它 物理量不变时, 熟化速率与界面张力成正比, 因而随超 声功率增加, 乳液的奥氏熟化速率增大, 表现为 $P=750$ $\mathrm{W}$ 时, 经长期静置的液滴直径最大. 而当 $P=1000 \mathrm{~W}$ 时, 其液滴直径随时间变化规律与奥氏熟化的拟合结果 偏离程度较大. 从图 $3 \mathrm{i} \sim 3 \mathrm{p}$ 含盐乳液静置 $168 \mathrm{~h}$ 后显微 照片分析可知, $P=1000 \mathrm{~W}$ 超声功率下乳液样品较其它 样品絮凝程度明显增大, 乳液絮凝促进了液滴之间发生 聚并, 这使得乳液的失稳方式变为聚并与奥氏熟化共同 作用. 

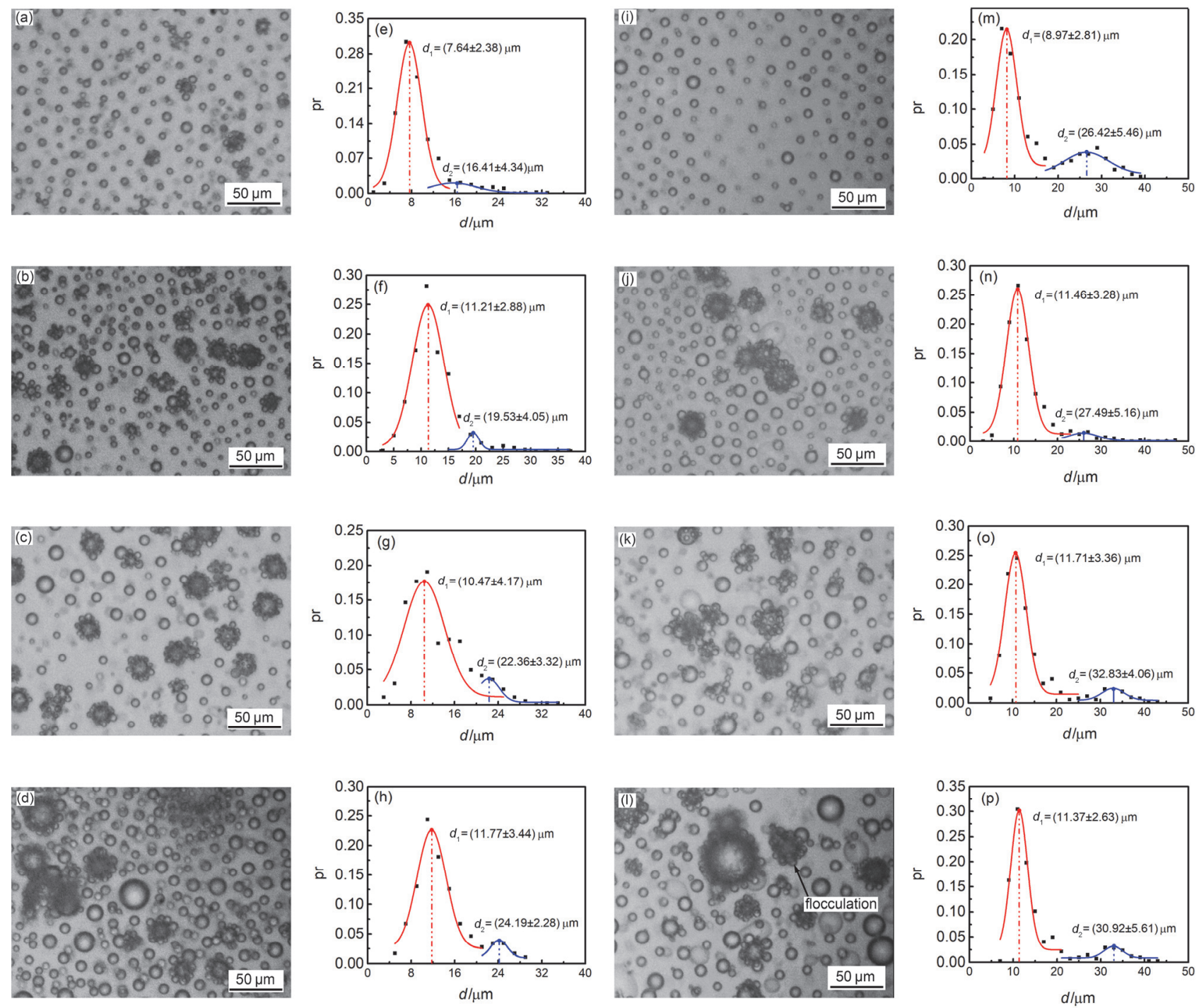

图 3 不同超声功率下制备的含盐乳液初始状态和静置 $168 \mathrm{~h}$ 后光学显微图和粒径分布图

Figure 3 Microscope images and droplets size distribution of emulsions at initial status and after $168 \mathrm{~h}$ initial status: (a, e) $250 \mathrm{~W}$; (b, f) $500 \mathrm{~W}$; (c, g) $750 \mathrm{~W}$; (d, h) $1000 \mathrm{~W}$. after 168 h: (i, m) $250 \mathrm{~W}$; (j, n) $500 \mathrm{~W}$; (k, o) $750 \mathrm{~W}$; (1, p) $1000 \mathrm{~W}$
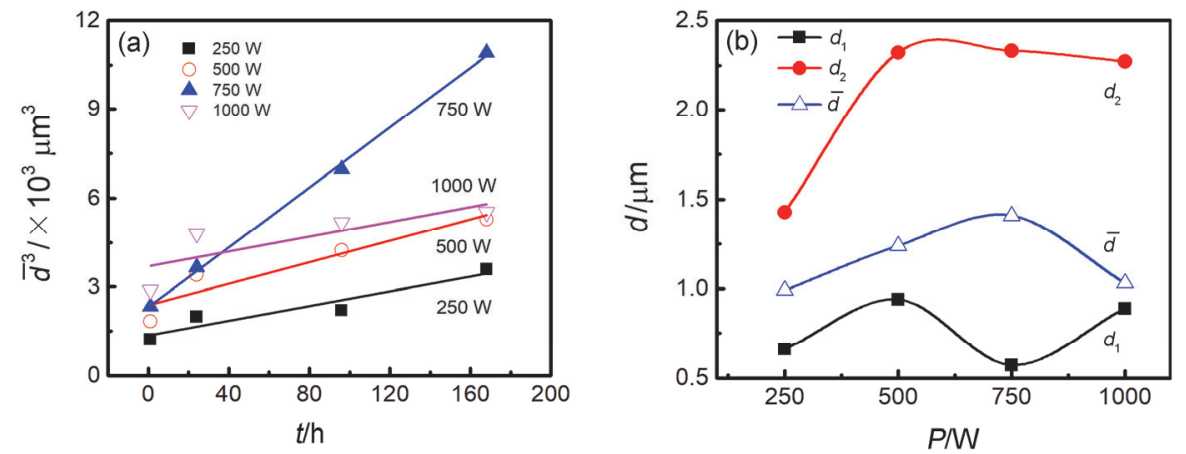

图 4 不同超声功率下分散相的形态特征

Figure 4 Configuration characteristics of dispersed phase under different ultrasonic powers

(a) the Ostwald ripening fitting curves of droplets size; (b) the average pore size of porous materials versus ultrasonic power

\section{3 超声乳化的含盐高分子乳液失稳机制}

乳液模板法制备多孔材料的最终结构与形貌, 与乳
液随时间推移过程中的失稳行为密切相关. 下文着重研 究乳液的稳定性与多孔结构的形貌之间的联系, 分析不 
同功率超声下制备的高分子乳液在玻璃纤维基底上制 备多孔材料的形貌, 并初步探索了超声功率-乳液失稳 机制一多孔结构形貌之间内在联系.

当四种超声功率下制备的含盐乳液静置 $168 \mathrm{~h}$ 后, 将玻璃纤维浸渍到每个乳液样品中, 然后取出并自然挥 干. 该方法可以通过超声功率调控乳液液滴粒径及分布 的手段, 以此调控 POSS-(PMMA) 8 为骨架的多孔材料的 孔径及分布, 以获得性质稳定、孔径可控的聚合物多孔 材料. 图 5 即为乳液静置 $168 \mathrm{~h}$ 后制备的多孔材料的 SEM 照片及对应每组多孔材料的孔径分布. 实验制备 的多孔材料结构高度有序, 形成开放的三维通道网络. 多孔材料的孔径与对应乳液的液滴直径相似, 分成两个 孔径分布区间.

图 $4 \mathrm{~b}$ 为多孔材料平均孔径随超声功率变化图, 从 图中可知, 不同超声功率下的多孔材料平均孔径变化规 律与对应乳液的液滴直径变化基本一致. 不同的是, 实 验制备的多孔材料孔径远小于对应含盐乳液的液滴直 径. 比较不同超声功率下的乳液在静置 $168 \mathrm{~h}$ 后制备的 多孔材料, 当超声功率较大和较小时, 多孔材料孔径较 小; 当超声功率适中时, 多孔材料孔径较大. 这是由于
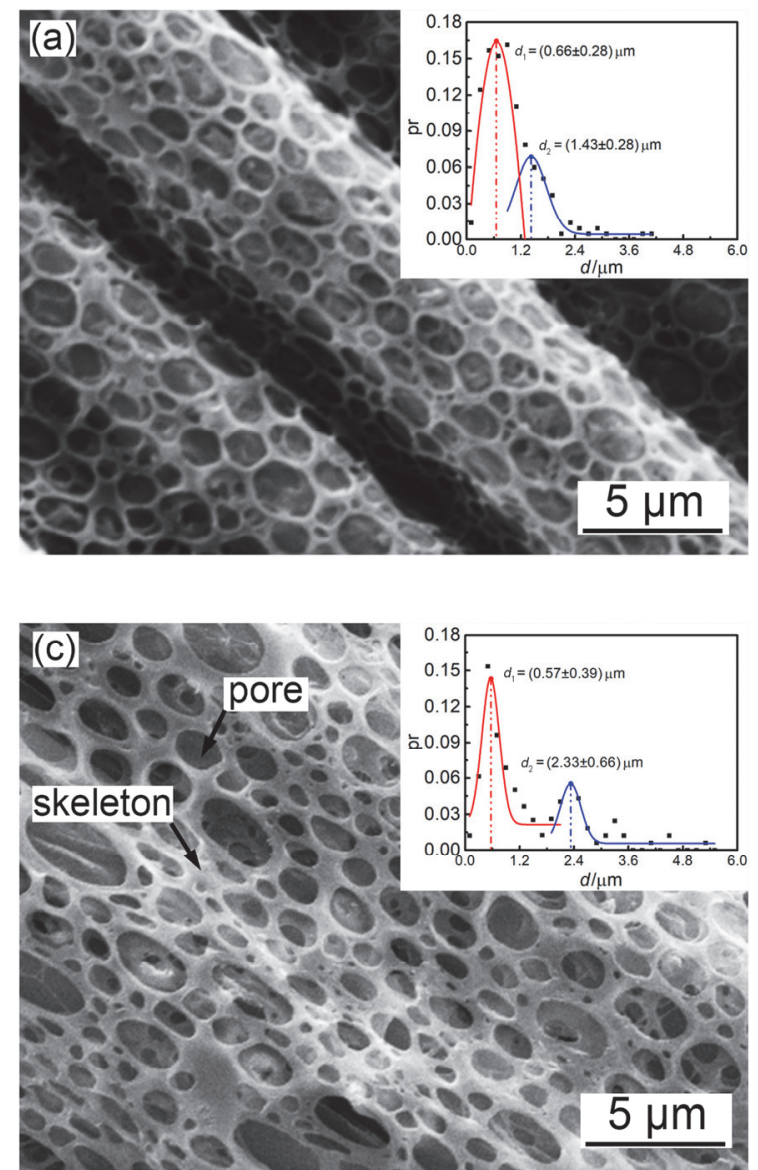

较大超声功率下 $(P=1000 \mathrm{~W})$ 制备的乳液在储存过程中, 其奥氏熟化受到聚合物分子絮凝的影响，导致溶剂挥发 后形成的多孔结构的孔径较小; 而超声功率适中时制备 的乳液失稳机制主要由奥氏熟化作用主导，且相比其它 较小超声功率条件下的乳液的奥氏熟化速率最快, 因此 溶剂挥发后获得的多孔结构孔径最大.

\section{3 结论}

本文研究了超声功率在超声乳化过程中对液滴直 径的调控机制，进一步分析通过乳液模板法制备多孔材 料的结构形貌，探索了超声功率-乳液失稳机制-多孔材 料形貌之间的联系, 得到以下结论.

(1) 超声作用下制备的高分子乳液，液滴直径存在 两个大小不同的直径下分布区间. 随超声功率的增加, 液滴直径随之增大; 超声场中制备的含盐乳液有絮凝现 象，且随超声功率的增加，含盐乳液絮凝程度增大.

(2) 超声功率在 $750 \mathrm{~W}$ 以下时, 含盐乳液的失稳方 式主要由奥氏熟化主导，且随超声功率增加，奥氏熟化 速率增大; 超声功率为 $1000 \mathrm{~W}$ 时, 由于分子絮凝程度 增大，乳液失稳由聚并和奥氏熟化共同作用.
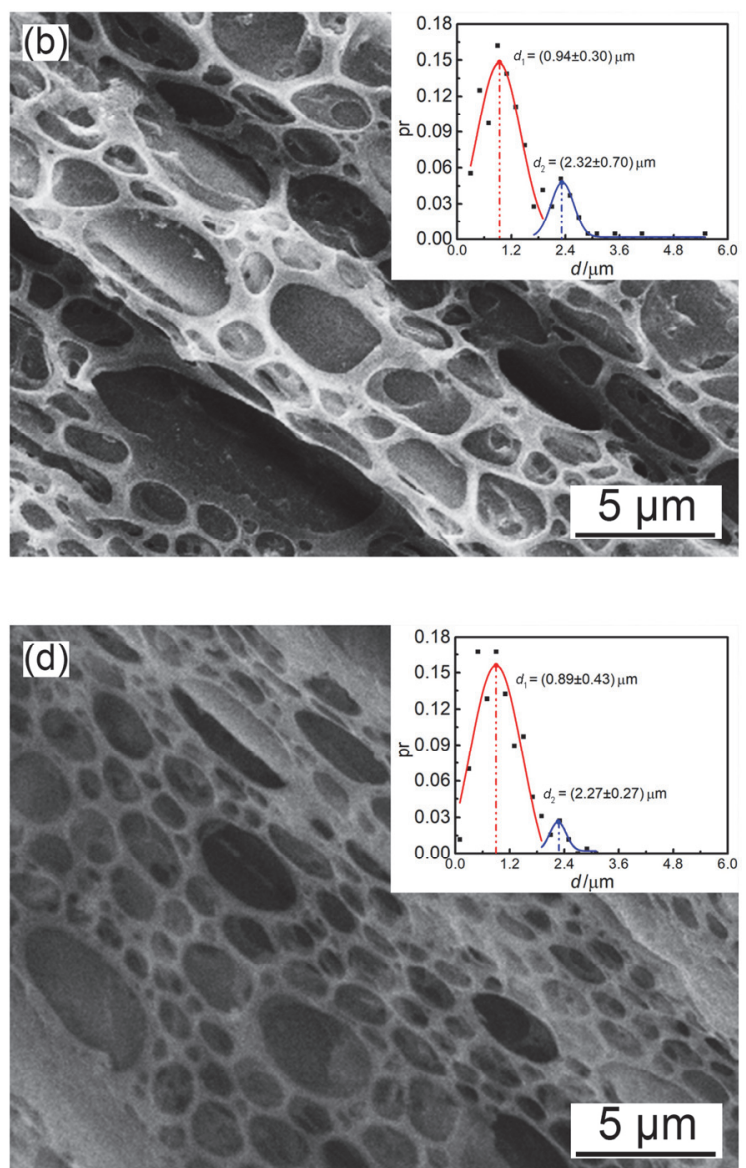

图 5 乳液在玻璃纤维基底上对应多孔材料的 SEM 图和多孔材料孔径分布图

Figure 5 SEM images and cavity size distribution of porous film on the glass fiber prepared by emulsions (a) $250 \mathrm{~W}$; (b) $500 \mathrm{~W}$; (c) $750 \mathrm{~W}$; (d) $1000 \mathrm{~W}$ 
(3) 以超声乳化的含盐乳液为模板制备的多孔材料 孔径与含盐乳液液滴直径的变化规律一致, 即利用超声 功率影响乳液失稳机制, 可制备孔径可调的多孔材料.

\section{4 实验部分}

实验过程中, 将等体积的油相(甲苯)和水相(纯水或 $0.1 \mathrm{~mol} \cdot \mathrm{L}^{-1}$ 的碳酸钠水溶液 $)$, 以及乳化剂 $\left[10 \mathrm{mg} \cdot \mathrm{mL}^{-1}\right.$ 溶于甲苯的大分子聚合物 POSS-(PMMA) 8 ] 置于烧杯中, 并将超声换能器变幅杆直接插入液相体系内部进行施 振, 以制备水油比 1: 1 的 w/o 型乳液. 所采用的超声功 率分别为 $P=250 、 500 、 750$ 和 $1000 \mathrm{~W}$, 超声振动时间 固定为 $5 \mathrm{~min}$. 乳液在静置 $1 、 24 、 96 、 168 \mathrm{~h}$ 后, 取少量 乳液滴在载玻片上并用适量甲苯稀释, 通过光学显微镜 观察液滴的微观形貌, 并使用 Image Pro Plus 软件测量 统计样品液滴的直径分布. 每组乳液至少在不同区域内 选取 500 个液滴的直径 ${ }^{[16]}$, 使数据具有更高的可靠性.

采用浸渍的方法, 将玻璃纤维插入在不同条件下制 备的含盐乳液中, 然后取出等待乳液溶剂自然挥发后得 到多孔结构. 通过 SEM 观察多孔材料的微观结构, 并统 计其孔径分布.

\section{References}

[1] (a) Quirk, R. A.; Trance, R. M.; Shakesheff, K. M.; Howdel, S. M. Curr. Opin. Solid State Mater. Sci. 2004, 8, 313. (b) Zhao, J. Y.; Zhu, Y. J.; Wang, Y. Y.; Zhu, J.; Shen, X. Chin. J. Org. Chem. 2017, 37, 203 (in Chinese). (赵俊颖, 朱艳吉, 王岩岩, 朱君, 沈筀, 有 机化学, 2017, 37, 203.) (c) Luo, H. L.; Sheng, J.; Wan, Y. Z. Mater. Lett. 2008, 62,37.

[2] (a) Wang, J. L.; Du, Z. J.; Li, H. Q.; Xiang, A. M.; Zhang, C. J. Colloid Interface Sci. 2009, 338, 145. (b) Ling, L.; Ching, W.; Vivian,
O. I.; Angelika, M. Macromol. Rapid Commun. 2011, 32, 1563. (c) Song, X. L.; You, J.; Xu, C. L.; Zhu, A. P.; Yan, C. F.; Guo, R. Chin. J. Chem. 2015, 33, 777 .

[3] Nie, L. H.; Tan, Q.; Zhu, W.; Wei, Q.; Lin, Z. K. Acta Phys.-Chim. Sin. 2015, 31, 1815 (in Chinese). (聂龙辉, 谭侨, 朱玮, 魏琪, 林 志奎, 物理化学学报, 2015, 31, 1815.)

[4] (a) Hu, Y.; Gu, X. Y.; Yang, Y.; Huang, J.; Hu, M.; Chen, W. K.; Tong, Z.; Wang, C. Y. ACS Appl. Mater. Interface 2014, 6, 17166. (b) Zheng, X. H.; Zhang, Y.; Wang, H. T.; Du, Q. G. Macromolecules 2014, 47, 6847. (c) Im, S. H.; Jeong, U. Y.; Xia, Y. N. Nat. Mater. $\mathbf{2 0 0 5}, 4,671$.

[5] (a) Liu, B.; Liu, J. G.; Liang, F. X.; Wang, Q.; Zhang, C. L.; Qu, X. Z.; Li, J. L.; Qiu, D.; Yang, Z. Z. Macromolecules 2012, 45, 5176. (b) Deng, W.; Chen, G.; Jia, L. K.; Wang, M. Y.; Gong, L. X. Acta Chim. Sinica. 2010, 68, 2000 (in Chinese). (邓伟, 陈国, 贾连昆, 王满意, 宫理想, 成友, 化学学报, 2010, 68, 2000.) (c) Zhai, W.; Liu, H. M.; Hong, Z. Y.; Xie, W. J.; Wei, B. B. Ultrason. Sonochem. 2017, 34, 130 .

[6] (a) Zhai, W.; Hong, Z. Y.; Wei, B. B. Sci. China. Ser. G 2007, 37, 367 (in Chinese). (翟薇, 洪振宇, 魏炳波, 中国科学: 物理学 力 学 天文学, 2007, 37, 367.) (b) Zhai, W.; Wang, B. J.; Liu, H. M.; Hu, L.; Wei, B. B. Sci. Rep. 2016, 6, 36718. (c) Rizzelli, S. L.; Jones, E. R.; Thompson, K. L.; Armes, S. R. Colloid Polym. Sci. 2016, 294, 1

[7] Sayin, C.; Ilhan, M.; Canakci, M.; Gumus, M. Renew. Energy. 2009, 34,1261

[8] Wei, X. L.; Wang, S. Y. J. Theor. Appl. Infor. Tech. 2013, 2, 1210.

[9] Sui, X. N.; Bi, S.; Qi, B. K.; Wang, Z. J.; Zhang, M.; Li, Y.; Jiang, L. Z. Food Hydrocolloids 2017, 63, 727.

[10] Leong, T. S. H.; Wooster, T. J.; Kentish, S. E.; Ashokkumar, M. Ultrason. Sonochem. 2009, 16, 721.

[11] Zhang, N.; Chen, G. J.; Chen, K.; Li, H. G.; Hao, J. C. Acta. Chim. Sinica 2015, 73, 835 (in Chinese). (张娜, 陈国君, 陈坤, 李洪光, 郝京诚, 化学学报, 2015, 73, 835.)

[12] Qiang, X.; Chen, F.; Ma, X. Y.; Hou, X. B. J. Appl. Polym. Sci. 2014, 131, 40652 .

[13] Taylor, G. I. Proc. R. Soc. London, A 1934, 858, 501.

[14] Liu, W. R.; Sun, D. J.; Li, C. F.; Liu, Q.; Xu, J. J. Colloid Interface Sci. 2006, 303, 557.

[15] Taylor, P. Adv. Colloid Interface Sci. 1998, 75, 107.

[16] Wu, T.; Wang, H. T.; Jing, B. X.; Liu, F.; Burns, P. C.; Na, C. Z. Nat. Commun. 2015, 6, 5259 . 